\title{
Predicting the cost of acute-care nursing: a nursing workload demonstration project I Daniel ${ }^{* 1}$, A Bandurchin ${ }^{2}$ and N Brooks ${ }^{2}$
}

\author{
Address: ${ }^{1}$ Health Policy Management and Evaluation, University of Toronto, Toronto, Ontario, Canada and ${ }^{2}$ Nursing Research Unit, University \\ Health Network, Toronto, Ontario, Canada \\ * Corresponding author
}

from 25th Patient Classification Systems International (PCSI) Working Conference

Fukuoka, Japan. II-I4 November 2009

Published: 5 November 2009

BMC Health Services Research 2009, 9(Suppl I):A20 doi:I0.II86/I472-6963-9-SI-A20

This abstract is available from: http://www.biomedcentral.com/I472-6963/9/SI/A20

(C) 2009 Daniel et al; licensee BioMed Central Ltd.

\section{Introduction}

Nursing workload data has not been used consistently for its original purpose: predicting staffing requirements and making staffing decisions. Too often, little time was paid to monitoring a workload measurement system to ensure that it accurately reflected the practice environment. Several studies have shown that workload measurement systems may not indicate true workload.

In 2006, the Ontario Ministry of Health and Long Term Care (MOHLTC) completed a study on the collection of nursing workload data. The study evaluated the quality and value of the data, and the cost-benefit of collecting the data. Results of the study led to the MOHLTC's recommending that the collection of nursing workload data be mandatory only for case-costing hospitals. The reason was that, at the time, there was no alternative in place to predict the cost of nursing.

The present nursing demonstration study, commissioned by the Nursing Secretariat of Ontario, Ministry of Health and Long Term Care, will explore the factors that affect nursing resources. It will also investigate the feasibility of a model to predict the use of nursing resources.

\section{Methods}

A time-and-motion study was conducted on three medi$\mathrm{cal} /$ surgical units in both an academic hospital and a large community hospital. Each staff nurse (RN, RPN, CA) on each unit was shadowed by a nursing student using a tablet computer, with custom-designed software, to capture the time spent by the nurses on 12 activities. Analyses were performed to investigate the amount of variation explained by the total nursing time spent on the nursing costs.

Using the hospital's administrative datasets, the costs of the resources consumed by patients, including nursing, supplies and medicine, were linked to the patients' nursing times recorded in the study. Regression analyses were done with the total nursing time as the independent variable, and the various patient costs as the dependent variables. Cost data included all resources consumed by the patient during his or her stay including nursing (estimated from workload data), supplies, diagnostics, therapies and overhead costs.

Administrative patient-level cost data was used from the case-costing dataset for 2006/07 for patients in the medi$\mathrm{cal}$, surgical and combined medical/surgical units in the two hospitals. Regression models were then explored to explain the variation in nursing costs. Various dependent cost variables were identified in the case-costing dataset. These included the following:

- Pharmacy costs

- Therapy costs

- Diagnostic imaging costs

- Clinical laboratory costs 
- Electrodiagnostic laboratory costs (e.g., EEG, EMG)

- Interventions (e.g., dialysis, endoscopy, DI intervention, cardiac catheterization lab)

- Intensive care unit costs

- Operating room costs

- Emergency department costs

- Clinic costs.

Age and acute-care length-of-stay were also included.

\section{Results}

Patient care accounted for the highest percentage (approximately 26\%) of nursing time, while average non-clinical time accounted for $20 \%$ of nursing time. The average amount of time spent on nursing activities varied by day of stay and by unit. $78.9 \%$ of the observed variance in direct nursing costs was explained by total nursing time spent $(\mathrm{p}<0.0001)$, as recorded in the time-and-motion study.

Using direct nursing cost data for medical, surgical and combined inpatient units for 2006/07, in the two hospitals, the costs of other services explained over $70 \%$ of the nursing cost variation. The variables selected were significant $(\mathrm{p}<0.001)$ and included length of stay, therapy costs, laboratory costs, diagnostic costs, ICU/CCU costs and pharmacy costs. Other variables, such as the costs of intervention, the emergency department, clinics and the operating room were significant. However, they increased the r-squared value very little.

\section{Conclusion}

This study confirmed that the nursing workload data from three wards in two hospitals correlated highly with timeand-motion study data. The simple model which was developed, using several factors, explained over $70 \%$ of nursing resource utilization. To enhance the model, future research will include the investigation of additional patient-level variables, as well as outcome variables (e.g., operating room times, discharge disposition, admission source, HOBIC scores, etc.).

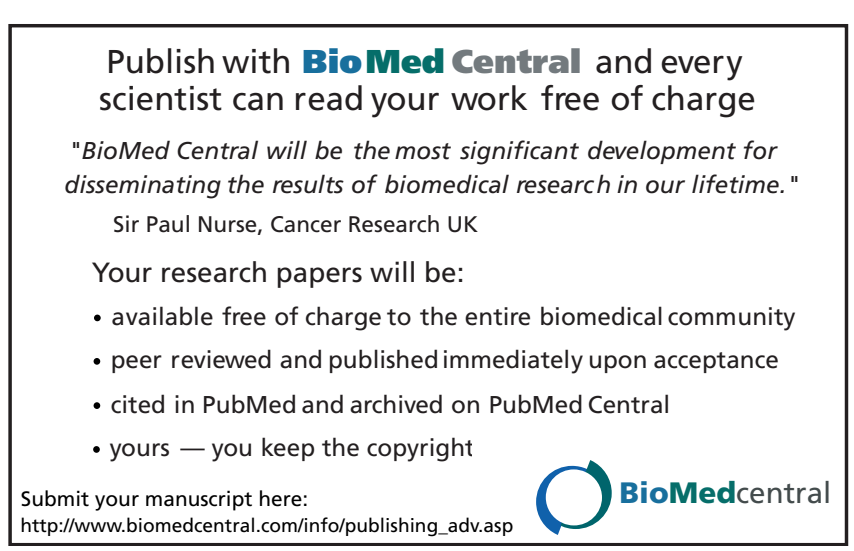

